\title{
Prediction of Heat Transfer For Turbulent Flow in Rotating Radial Duct
}

\author{
P. TEKRIWAL \\ GE Corporate Research \& Development, Schenectady, NY 12301
}

(Received August 9, 1995)

\begin{abstract}
The objective of the current modeling effort is to validate the numerical model and improve upon the prediction of heat transfer in rotating systems. Low-Reynolds number turbulence model (without the wall function) has been employed for three-dimensional heat transfer predictions for radially outward flow in a square cooling duct rotating about an axis perpendicular to its length. Computations are also made using the standard and extended high-Reynolds number $\mathrm{k}-\epsilon$ turbulence models (in conjunction with the wall function) for the same flow configuration. The results from all these models are compared with experimental data for flows at different rotation numbers and Reynolds number equal to 25,000 . The results show that the low-Reynolds number model predictions are not as good as the high-Re model predictions with the wall function. The wall function formulation predicts the right trend of heat transfer profile and the agreement with the data is within $30 \%$ or so for flows at high rotation number. Since the Navier-Stokes equations are integrated all the way to wall in the case of low-Re model, the computation time is relatively high and the convergence is rather slow, thus rendering the low-Re model as an unattractive choice for rotating flows at high Reynolds number.

The extended $k-\epsilon$ turbulence model is also employed to compute heat transfer for rotating flows with uneven wall temperatures and uniform wall heat flux conditions. The comparison with the experimental data available in literature shows that the predictions on both the leading wall and the trailing wall are satisfactory and within 5-25\% agreement.
\end{abstract}

Key Words: Low-Re turbulence model; $k-\epsilon$ turbulence model; Rotating Machinery; Heat transfer; Uneven wall temperature

\section{INTRODUCTION}

$\mathrm{O}$ ne of the methods through which cooling of modern gas turbine blades is achieved is to provide the gas flow through internal ducts of the blades. These ducts can vary in shape and size as shown in Figure 1. For an empirical design a large experimental data base is required because the geometry and flow conditions can vary in a wide range. An analytical tool obviously can substitute for experimental work and reduce the cost of design. Any analytical tool, however, has to be validated with experimental data before it can be reliably used. The current effort deals with validation of the numerical model for heat transfer predictions in rotating machinery. The flow considered is radially outward through a single square duct rotating in orthogonal mode.

Several contributions exist in literature for the kind of flow considered here and an extensive review was reported by the author in an earlier work [1992a]. These included experimental as well as numerical work for flow and heat transfer in smooth rotating radial ducts and with some exceptions will not be repeated here. Recently, Yang et al. [1992b] obtained heat transfer data for rotating serpentine square flow passage for rotation number up to 0.042 . They showed that the effect of rotation is seen locally on the leading, trailing and side walls whereas little effect is seen on the circumferentially averaged Nusselt number due to rotation. Heat transfer increases with increasing Reynolds number and the effect of buoyancy on heat transfer exists in the inlet region and diminishes downstream. Heat transfer is significantly enhanced at sharp turns due to secondary flow. Han and Zhang [1992c] experimentally studied the effect of uneven wall temperatures on heat transfer in a square duct for rotating radial outflow for a range of Re from 2500 to 25000 and rotation number up to 0.352 . Data for the case of uniform wall heat flux were also obtained. The heat transfer for the trailing surface in the uniform 


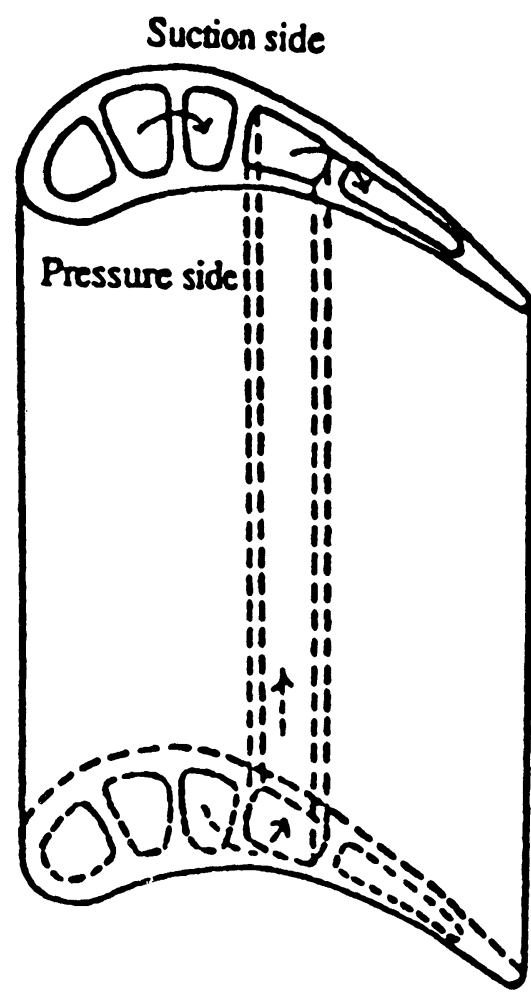

FIGURE 1 Internal cooling passages in a turbine blade.

wall heat flux case is $10-20 \%$ higher than in the case of uniform wall temperature. The heat transfer on the leading wall is higher by $40-80 \%$ in the case of uniform wall heat flux and by $20-40 \%$ in the case of uneven wall temperature when compared with heat transfer in the case of isothermal walls. The author [1992a] employed the standard and extended $\mathrm{k}-\epsilon$ turbulence models in conjunction with wall function to predict heat transfer in single-pass rotating cooling channel and compared the results with experimental data of Wagner et al. [1989] for square duct and the data of Morris and Ghavami-Nasr [1990] for rectangular duct. Effects of Coriolis force, centrifugal force and the centrifugal buoyancy were included and discussed in detail. The extended k- $\epsilon$ model was found to improve the heat transfer predictions by about $15 \%$ compared to the standard k- $\epsilon$ model at high rotation number flows (Ro $>0.2$ ). The purpose of the current effort is two-fold. First, the low-Re k- $\epsilon$ turbulence model is employed in which the Navier-Stokes equations are integrated all the way to the wall (no wall function used) and after comparing the results with the experimental data it is determined if the predictions benefit from the use of the low-Re model. The results of standard and extended $\mathrm{k}-\epsilon$ models with wall function are also shown on the same graph to provide a comparative assessment of these models. The second purpose is to apply the turbulence model for the case of uneven wall temperatures and judge the performance by comparing with the data. The low-Re model chosen for the current work is that of Lam and Bremhorst [1981] as this has been known to be one of the reliable models. (See Patel et al. [1985a] and Biswas and Fukuyama [1993]).

\section{GEOMETRY DESCRIPTION}

Figure 2 shows a rectangular duct of dimension $d \times d \times$ $\mathrm{L}$ rotating at a constant angular speed $\omega$ about $\mathrm{X}$-axis. Flow of the coolant (air) is radially outward as shown. The eccentricity or the root radius of the duct is given as $R_{r}$ and the mid-height of the duct is denoted by $R$. Geometry and the flow conditions are symmetric about the longitudinal mid-plane of the duct, and therefore, flow in only one-half of the duct is discretized for computational economy.

As shown in Figure 2, Coriolis forces produce a secondary flow in cross-stream planes which causes the migration of flow from the central core region to the trailing wall of the duct with a return flow along the walls in order to maintain continuity. As a result, two symmetric vortices are formed in the planes perpendicular to the main flow direction. This secondary flow leads to an increase in wall friction and heat transfer at the trailing wall in comparison to those at the leading wall.

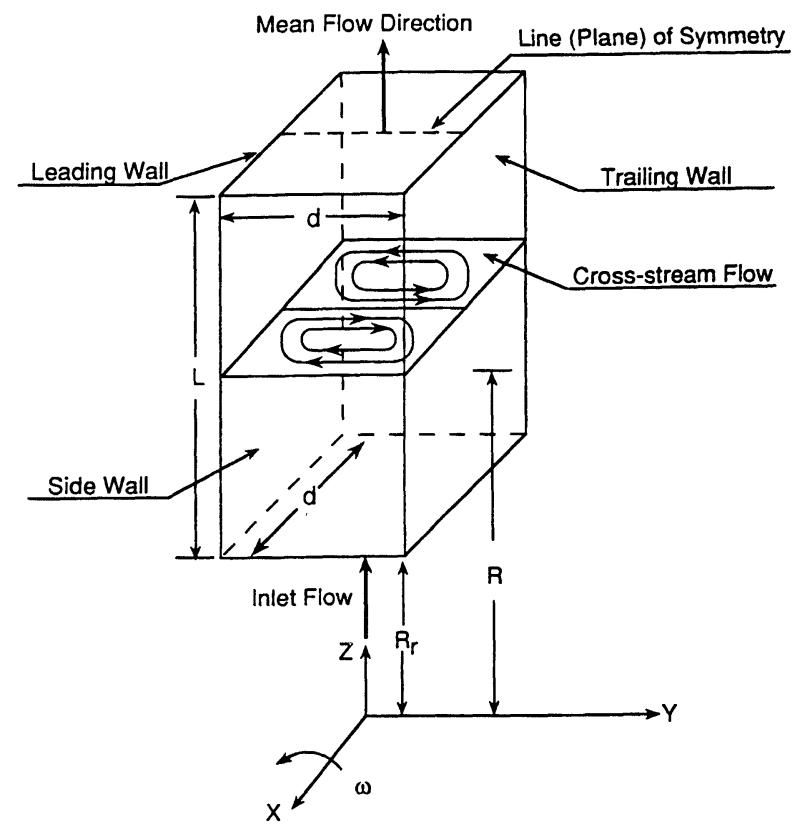

FIGURE 2 Illustration of flow geometry. 
In the case of heated walls, a temperature gradient exists from the wall region to the core which gives rise to centrifugal buoyancy. In the case of radially outward duct flow, the radial velocity of the relatively cooler, and hence heavier, fluid near the trailing wall is increased while the radial velocity of the relatively warmer, and hence lighter, fluid near the leading wall is decreased. Consequently, the friction and heat transfer near the trailing wall are further enhanced [1992a].

\section{TURBULENCE MODELS}

The details of the Navier-Stokes equation governing the flow and the details of the $k-\epsilon$ turbulence models have been provided by the author in Ref. [1992a] and will not be repeated here. The low-Re version of $k-\epsilon$ turbulence model is described here. The purpose of a turbulence model is to determine the turbulent viscosity $\left(\mu_{t}\right)$ so that the system of equations can be closed in mathematical sense.

The turbulent viscosity is obtained from the following set of equations [1981]:

$$
\begin{gathered}
\mu_{t}=\mathrm{C}_{\mu} f_{\mu} \rho k^{2} / \epsilon \\
(\underset{\sim}{\mathrm{w}} \cdot \underset{\sim}{\nabla}) \rho \mathrm{k}=\underset{\sim}{\nabla}\left(\frac{\mu_{\mathrm{t}}}{\mathrm{Pr}_{\mathrm{k}}} \underset{\sim}{\nabla \mathrm{k}}\right)+\rho\left(\mathrm{G}_{\mathrm{k}}-\epsilon\right) \\
(\underset{\sim}{\mathrm{w}} \cdot \underset{\sim}{\nabla}) \rho \epsilon=\underset{\sim}{\nabla}\left(\frac{\mu_{\mathrm{t}}}{\operatorname{Pr}_{\epsilon}} \underset{\sim}{\nabla}\right)+\mathrm{C}_{1} \mathrm{f}_{1} \rho \mathrm{G}_{\mathrm{k}} \frac{\epsilon}{\mathrm{k}}-\mathrm{C}_{2} \mathrm{f}_{2} \rho \frac{\epsilon^{2}}{\mathrm{k}}
\end{gathered}
$$

where the production term $G_{k}$ is given by

$\mathrm{G}_{k}=\frac{\mu_{t}}{\rho}\left(\frac{\partial w_{i}}{\partial x_{j}}+\frac{\partial w_{j}}{\partial x_{i}}\right) \frac{\partial w_{i}}{\partial x_{j}}$ (summation convention implied)

The constants in the equations used are as follows:

$$
\begin{aligned}
\mathrm{C}_{\mu} & =0.09, \mathrm{Pr}_{k}=1.0, \mathrm{Pr}_{\epsilon}=1.3, \mathrm{C}_{1} \\
& =1.44 \text { and } \mathrm{C}_{2}=1.92
\end{aligned}
$$

The functions $f_{\mu}, f_{1}$ and $f_{2}$ are computed as

$$
\begin{gathered}
f_{\mu}=\left(1-e^{-0.0165 \mathrm{R}_{k}}\right)^{2}\left(1+20.5 / \mathrm{R}_{t}\right) \\
f_{1}=1+\left(\frac{0.05}{f_{\mu}}\right)^{3}
\end{gathered}
$$

$$
f_{2}=1-e^{-\mathrm{R}_{t}^{2}}
$$

where,

$$
\mathrm{R}_{k}=k^{1 / 2} y \rho / \mu \text { and } R_{t}=k^{2} \rho / \mu \epsilon
$$

It should be noted that by introducing the "near-wall functions" $f_{\mu}, f_{1}$ and $f_{2}$ the general form of the 2-equation $\mathrm{k}-\epsilon$ model for high-Re flow has been retained. In the regions far away from the wall where the flow is fully turbulent, these functions assume the value of unity thus conforming to the standard high-Re version of the $\mathrm{k}-\epsilon$ model.

The boundary conditions for $\mathrm{k}$ and $\epsilon$ at the wall remain to be specified. At a wall $\mathrm{k}=0$ and $\epsilon$ is finite [1981]. Zero gradient boundary condition $(\partial \epsilon / \partial y=0)$ for the dissipation rate is specified at the wall [1985a]. An alternative boundary condition for $\epsilon$ at the wall $(\epsilon=$ $\left.2 \mu \mathrm{k}_{\mathrm{p}} / \delta^{2} \rho\right)$ yields almost identical results for the case considered in the present paper.

\section{NUMERICAL SOLUTION}

The fully elliptic 3-D conservation equations are integrated over control volumes and finite difference approximations are obtained by discretizing them. The staggered grid approach described by Patankar [1980] is used. The scaler variables ( $\mathrm{p}, \mathrm{k}, \epsilon, \mathrm{H}, \rho$ etc.) are stored at the center of control volumes and the velocity components are located at the control volume faces. The equations are solved by SIMPLE algorithm extended to the curvilinear coordinate system. The detailed implementation of the numerical algorithms is given by 3) Braaten and Shyy [1986] and Shyy et al. [1985b]. The hybrid interpolating scheme has been used for the computation.

Unless specified otherwise, number of control volume cells used in the symmetric half-duct (see Figure 2) is 10 $\times 20 \times 50$ for the solution using wall function. 10000 cells were determined to be adequate for the present square duct in the previous work [1992a]. For the low-Re model numerical experiment was done on the grid size variation to achieve grid-independent solution. A mesh of $20 \times 40 \times 100$ cells was chosen for most of low-Re computations. The cell size is decreased as it gets closer to the walls, with the ratio of cross-stream plane areas between two adjacent cells not dropping below 0.83 .

\section{RESULTS AND DISCUSSION}

The geometrical dimensions of the square duct of Wagner et al. [1989] and Han and Zhang [1992c] are given in 
TABLE 1

Geometrical Dimensions of the Ducts

\begin{tabular}{lcc}
\hline Parameter & Wagner, et al. $[4]$ & Han and Zhang [3] \\
\hline $\mathrm{d}(\mathrm{cm})$ & 1.27 & 1.27 \\
$\mathrm{~L}(\mathrm{~cm})$ & 18.16 & 15.24 \\
$\mathrm{R}_{\mathrm{r}}(\mathrm{cm})$ & 53.21 & 30.48 \\
\hline
\end{tabular}

Table 1. The heat transfer results are presented in the form of radial $(\mathrm{z} / \mathrm{d})$ variation of the Nusselt number ratio $\left(\mathrm{Nu} / \mathrm{Nu}_{\infty}\right)$ on the trailing and leading walls where $\mathrm{z}$ is the distance from the inlet section and $\mathrm{Nu}_{\infty}$ is the Nusselt number for a fully developed flow in a non-rotating duct. $\mathrm{Nu}_{\infty}$ is calculated as $0.022 \mathrm{Re}^{0.8} \operatorname{Pr}^{0.6}$ for the case of comparison with Wagner's data [1989] and $0.023 \mathrm{Re}^{0.8}$ $\mathrm{Pr}^{0.4}$ for the case of comparison with Han and Zhang's data [1992c]. Pr value used for air is 0.72 . The density is calculated by using the ideal gas law. Reynolds number calculation is based on average velocity at the inlet. The velocity profile used at the inlet is the fully turbulent $1 / 7$ th power profile given as

$$
w_{z}=w_{c}(1-2|x| / d)^{1 / 7}(1-2|y| / d)^{1 / 7}
$$

\section{Grid-size Effect and Convergence}

Resolution of the near wall region is an important issue in a low-Re calculation. Low-Re solutions were obtained for three different grid densities and they are shown in Figures 3 and 4 for the trailing and leading wall respectively. The data points from Wagner et al. [1989] are also plotted in Figures 3 and 4 for comparison even though comparison of model results with the experimental data is discussed in more detail through Figures 5 through 8 . Number of cells for these three meshes are 16 $\times 32 \times 100(=51200), 20 \times 40 \times 100(=80000)$ and 24 $\times 48 \times 100(=115200)$ in the symmetric half duct of Wagner et al. [1989]. The resulting $\mathrm{y}^{+}$values were in the range 4-15, 3-8 and 1.5-7 respectively. As we can see in Figure 3, the Nusselt number ratio for the coarse grid (51200 cells) is about $15-20 \%$ lower (percent with respect to data) than the medium (80000 cells) and fine $(115,200$ cells) grid solutions in the range of $z / d$ equal to $4-8$. Prior to $\mathrm{z} / \mathrm{d}$ equal to 8 , the medium and fine grid results are close to each other. Beyond $z / 8=8$, the coarse and medium grid results are close to each other and the fine grid solution is slightly higher. For the numerical results on the leading wall in Figure 4, we notice that the coarse grid results are substantially lower (by up to $25 \%$ with respect to the calculated low-Re result) than the results from the medium size and fine size

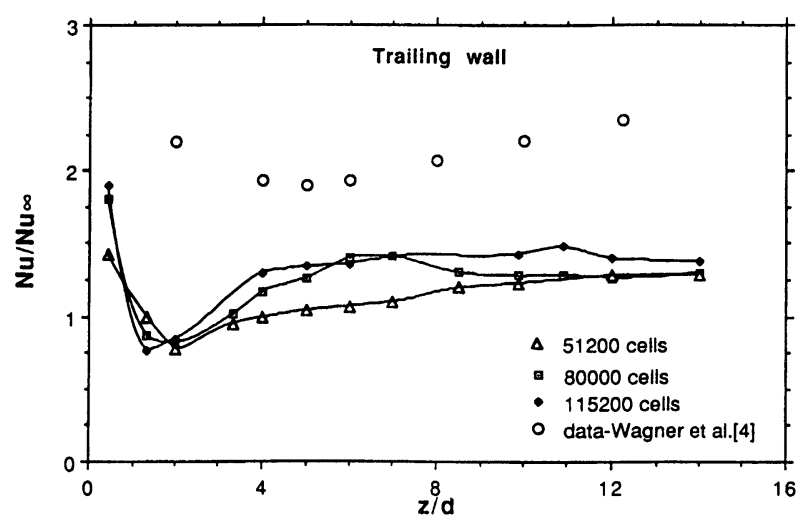

FIGURE 3 Grid size effect on low-Re calculations $(\mathrm{Ro}=.24, \mathrm{Re}=$ $25000, \Delta \mathrm{T} / \mathrm{Tw}=.13$ ).

grids. The results from the medium and fine grids are relatively close to each other except near $z / d=11$. It is concluded from the numerical results shown in Figures 3 and 4 that a reasonable grid-independent solution can be obtained with $20 \times 40 \times 100$ cells for flow configuration considered here. The low-Re results reported in Figures 5 through 8 are based on a mesh of $20 \times 40 \times 100$ cells.

The convergence rate in the case of low-Re calculations is much slower than in the case of high-Re model with wall function. In order to obtain a mass residual of the order of $10^{-3}$, the wall function calculation required approximately 600 iterations whereas a typical low-Re calculation needed 5000-8000 iterations. Since the mesh size in a low-Re computation is 8 times larger than the mesh in a wall function calculation, each iteration in a low-Re calculation is more expensive. As an example on a CONVEX computer, a high-Re (with wall function)

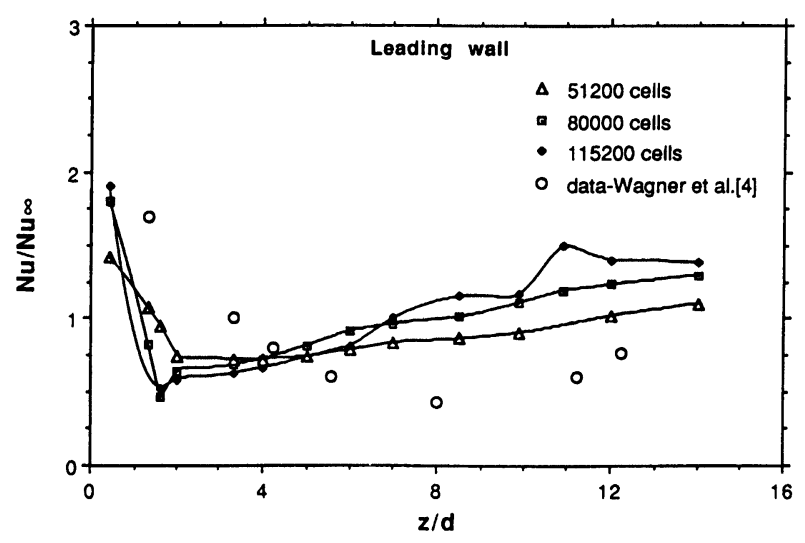

FIGURE 4 Grid size effect on low-Re calculations $(\mathrm{Ro}=.24, \mathrm{Re}=$ $25000, \Delta \mathrm{T} / \mathrm{Tw}=.13)$. 


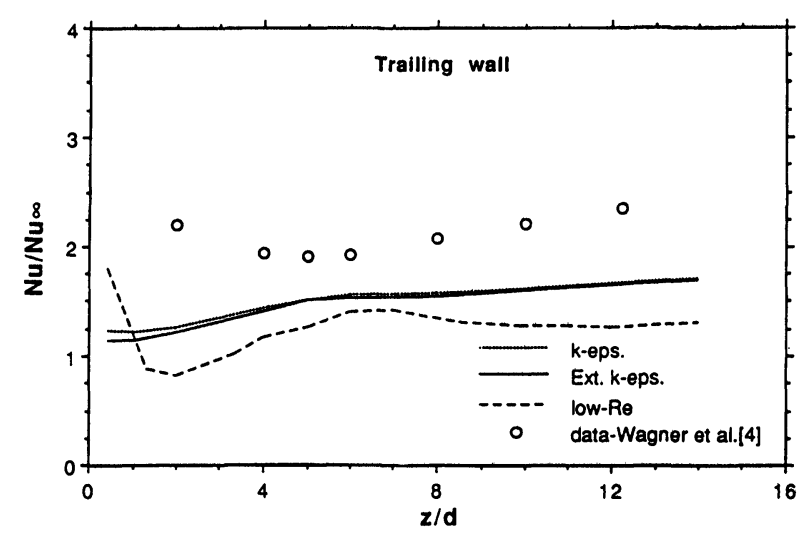

FIGURE 5 Comparison of model results with data $(\operatorname{Ro}=.24, \mathrm{Re}=$ $25000, \Delta \mathrm{T} / \mathrm{Tw}=.13)$.

calculation required $5.3 \times 10^{3} \mathrm{cpu}$ seconds for 1500 iterations on a 10000 -cell mesh to yield a mass residual of $10^{-4}$ whereas a low-Re calculation took $1.2 \times 10^{5} \mathrm{cpu}$ seconds for 2000 iterations on an 80000-cell mesh to yield a mass residual of $10^{-2}$. The residual is defined as the ratio of sum of the residuals of all the cells to the total quantity at the inlet.

\section{Comparison of Different Model Results with Data}

Figures 5 and 6 compare the high-Re (standard $k-\epsilon$ and extended $\mathrm{k}-\epsilon$ models with wall function) and low-Re results with the data for the trailing wall and the leading wall respectively at $\operatorname{Ro}=0.24$ and $\operatorname{Re}=25000$. In Figure 5 the two high-Re versions of $k-\epsilon$ turbulence models yield almost identical results. Except near the inlet, the maximum difference between the data and the high-Re predictions is $26 \%$ (with respect to the data)

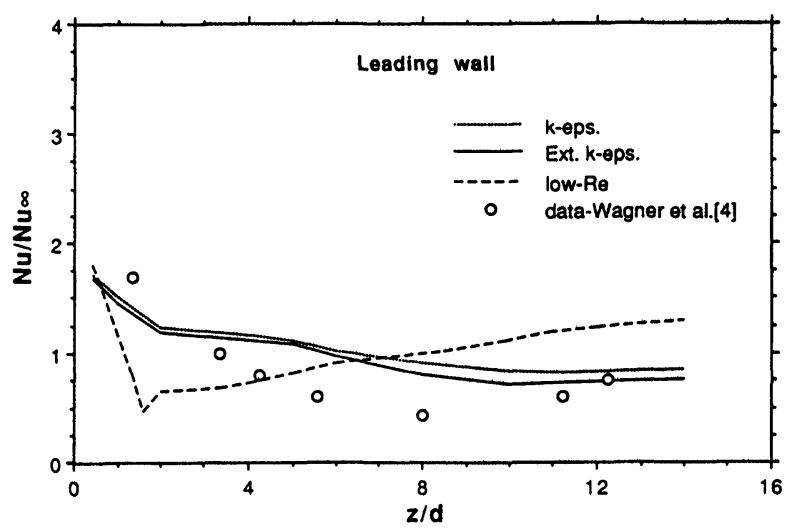

FIGURE 6 Comparison of model results with data $(\operatorname{Ro}=.24, \mathrm{Re}=$ $25000, \Delta \mathrm{T} / \mathrm{Tw}=.13$ ).

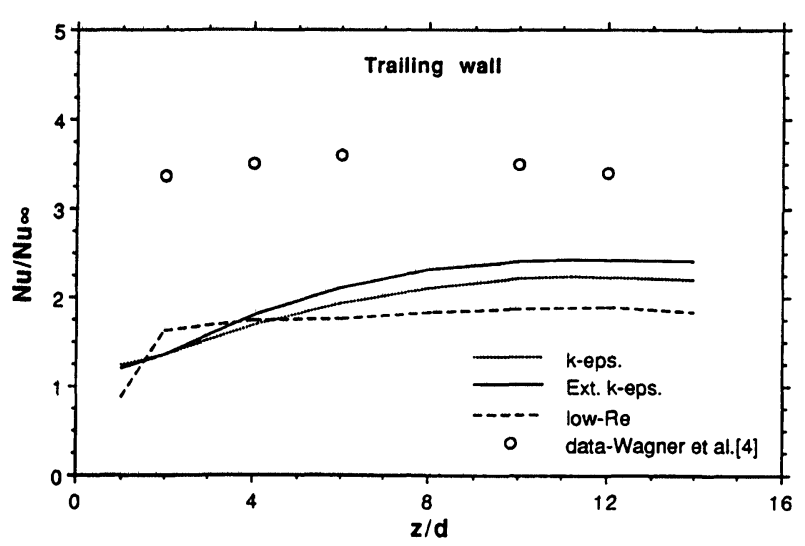

FIGURE 7 Comparison of model results with data $(\mathrm{Ro}=.48, \mathrm{Re}=$ $25000, \Delta \mathrm{T} / \mathrm{Tw}=.13$ ).

whereas the maximum difference between the low-Re prediction and the data is about $48 \%$. The low-Re prediction trails behind the high-Re prediction at all the axial locations. The high-Re model predictions show the trend of increasing heat transfer after $z / d=6$ or so as noticed in the data also, whereas the low-Re prediction fails to do so. However, the rising trend in the $\mathrm{Nu} / \mathrm{Nu}_{\infty}$ from high-Re predictions is not as steep as seen in the data. The data for the leading wall in Figure 6 show a decreasing trend from inlet region to $z / d$ equal to about 8 and then a rising trend till near the exit. The high-Re model results also show the declining trend from inlet region to $\mathrm{z} / \mathrm{d}$ equal to nearly 10 and then an upward turn till near the exit. The low-Re results, however show a poor performance when compared with the data.

Figures 7 and 8 compare different model performances at a higher rotation number $(\mathrm{Ro}=0.48)$. In the inlet region the numerical results have a relatively large

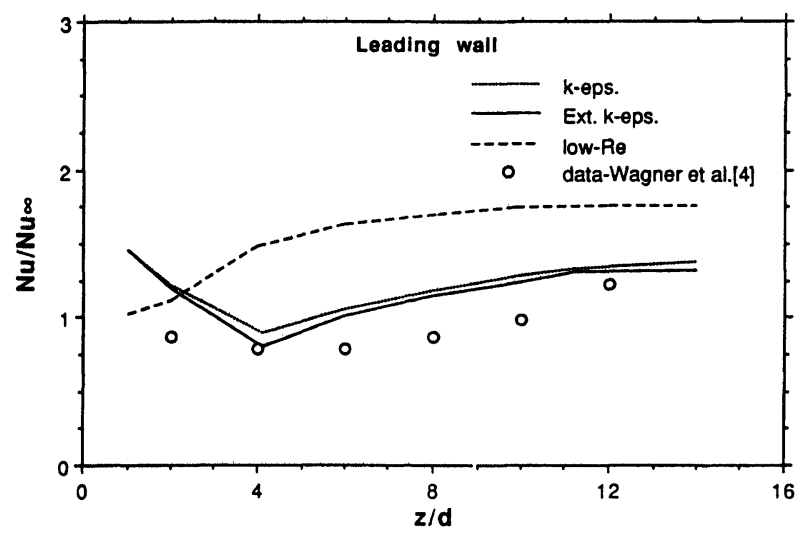

FIGURE 8 Comparison of model results with data $(\mathrm{Ro}=.48, \mathrm{Re}=$ $25000, \Delta \mathrm{T} / \mathrm{Tw}=.13$ ). 


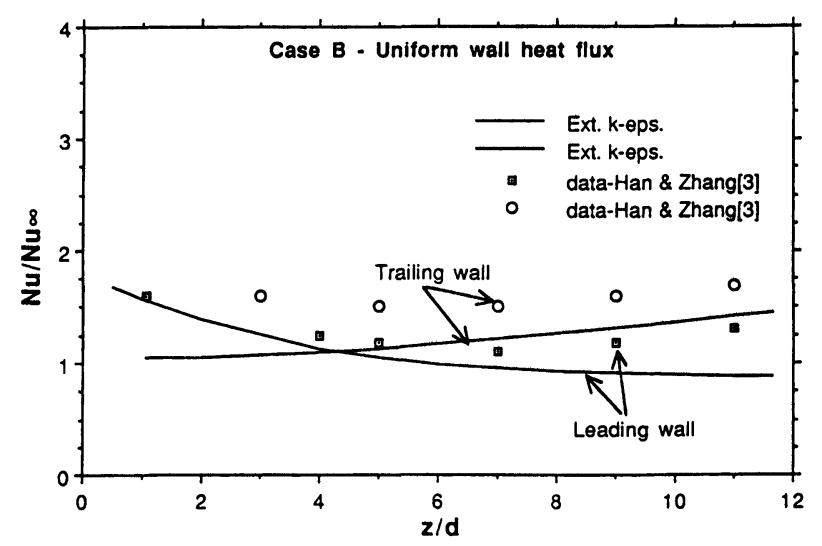

FIGURE 9 Comparison for uniform wall heat flux case ( $\mathrm{Ro}=.035$, $\operatorname{Re}=25000)$.

difference with the data because of the neglect of swirl in the flow at the inlet, inability of wall function to predict heat transfer in the high gradient region and the experimental error in data [1992a]. In Figures 7 and 8 both, we note that the high-Re models in conjunction with wall function perform better than the low-Re turbulence model in predicting the heat transfer. Among the two versions of high-Re model, the extended $\mathrm{k}-\epsilon$ model predictions are closer to the data by $5-15 \%$ or so. The maximum difference between the data and the extended $\mathrm{k}-\epsilon$ model prediction is about $37 \%$ at $\mathrm{z} / \mathrm{d}=6$ in Figure 7. The low-Re model performance seems to be "poor and unacceptable" in comparison to the high-Re model in Figure 8.

\section{Uneven Wall Temperatures}

In all the cases discussed so far all the walls were at uniform common temperature $\left(\mathrm{T}_{\mathrm{w}}\right)$ and the extended high-Re k- $\epsilon$ model along with wall function predicts heat

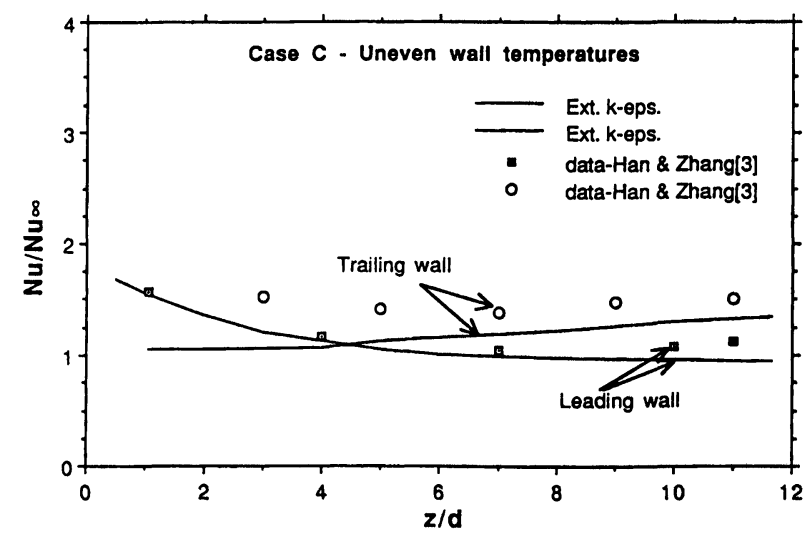

FIGURE 10 Comparison for uneven wall temperatures $(\mathrm{Ro}=.035$, $\operatorname{Re}=25000)$.

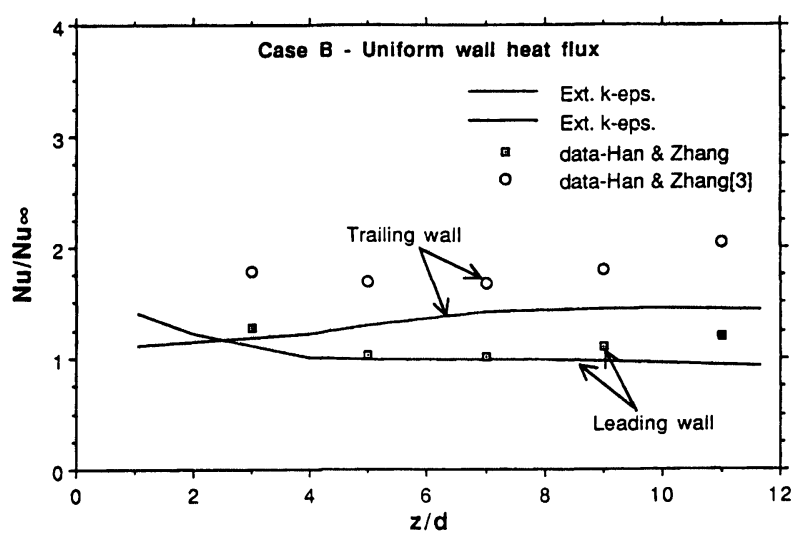

FIGURE 11 Comparison for uniform wall heat flux $(\mathrm{Ro}=.088, \mathrm{Re}$ $=10000)$

transfer reasonably satisfactorily. In the remaining section we employ the extended $k-\epsilon$ model for a similar flow configuration but with uneven wall temperatures. Recently published data of Han and Zhang [1992c] are used to compare the model results. There are two different cases of boundary conditions-case B and case C as termed by Han and Zhang [1992c]. Case B represents a uniform wall heat flux condition and case $\mathrm{C}$ represents an uneven wall temperature condition. The uniform wall heat flux for case B at Ro $=0.035$ is $6172 \mathrm{~W} / \mathrm{m}^{2}$ and at Ro $=0.088$ is $2916 \mathrm{~W} / \mathrm{m}^{2}$. For case $\mathrm{C}$, the leading and trailing walls are at $62.5^{\circ} \mathrm{C}$ and the side walls are at $50^{\circ} \mathrm{C}$. The inlet temperature in all the cases is $30^{\circ} \mathrm{C}$.

Figure 9 shows the numerical results and the experimental data for both the trailing and leading walls for a flow at $\mathrm{Ro}=0.035$ and $\mathrm{Re}=25000$ with boundary condition of case $\mathrm{B}$. The numerical model predictions of Nusselt number ratio are within $25 \%$ of the data except near the inlet at the trailing wall. The experimental rig has an entrance region to establish hydrodynamically

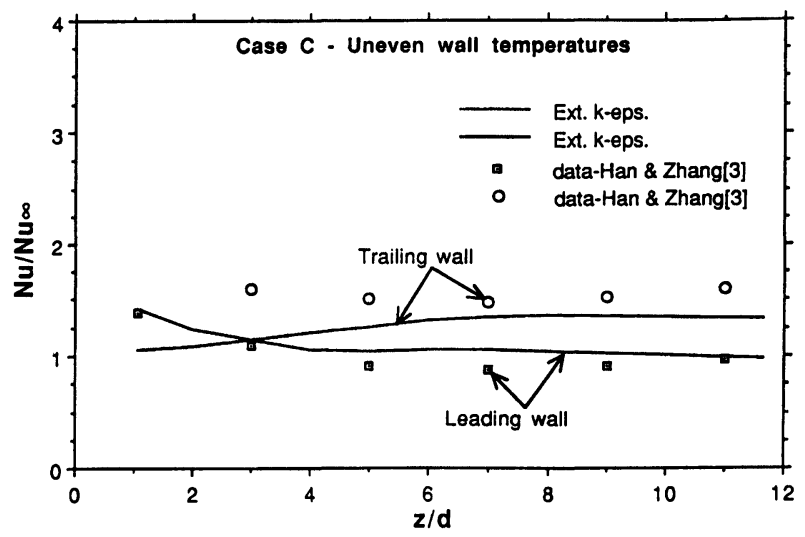

FIGURE 12 Comparison for uneven wall temperatures ( $R=.088$, $\operatorname{Re}=10000)$ 
fully developed flow at the entrance to the test duct [1992c]. This entrance region is not modeled in the numerical simulation which will introduce discrepancy in the results. Figure 10 shows the results and the data for case $C$ condition, $\mathrm{Ro}=0.035$ and $\operatorname{Re}=25000$. The agreement of numerical predictions with the data is quite satisfactory except near the inlet at the trailing wall.

Figures 11 and 12 compare the model results for case $\mathrm{B}$ and case $\mathrm{C}$ boundary conditions at $\mathrm{Ro}=0.088$ and $\mathrm{Re}$ $=10000$. The agreement of the numerical predictions in both the cases is once again within $25 \%$ of the data at the trailing as well as the leading wall. The only noticeable difference between the results in Figure 12 and Figures 9 through 11 is that the data for the leading wall are overpredicted in Figure 12 whereas in all other cases (Figures 9-12 only) the data are underpredicted. However, the difference between the data and predictions for the leading wall are well within the bounds of uncertainty of experimental measurements which could be up to $20-25 \%$ [1992c].

\section{CONCLUSIONS}

1. The low-Reynolds number model utilizes a large number of cells and the convergence rate is very slow in comparison to the high-Reynolds number model using wall function. It is difficult and expensive to obtain a well converged solution with the low-Re turbulence model.

2. The poor agreement of the low-Re model results with the data makes the low-Re model as an unattractive choice for heat transfer computations in rotating radial outward flow at high Rotation number $(>0.24)$ and high-Reynolds number (25000).

3. The extended version of high-Reynolds number turbulence model in conjunction with wall function yields satisfactory results for flows with isothermal walls as well as uneven wall temperatures. The agreement is within $5-25 \%$ of the data with uneven wall temperatures. However, the Reynolds numbers considered in the present work were 25000 and 10000. A flow at lower Reynolds number may prevent the use of wall function because the $\mathrm{y}^{+}$value for the near wall cells may drop below the logarithmic velocity profile regime.

\footnotetext{
Nomenclature

$C_{l}, C_{2}, C_{\mu}$

$d$

$f_{\mu} f_{1}, f_{2}$
}

Turbulence model constants

Hydraulic diameter

Low-Re turbulence model functions

$\begin{array}{ll}G_{k} & \text { Production rate of kinetic energy } \\ H & \text { Enthalpy } \\ h & \text { Heat transfer coefficient } \\ k & \text { Turbulent kinetic energy } \\ k_{p} & \text { k at near-wall cell } \\ L & \text { Duct length } \\ N u & \text { Nusselt number, hd } / \lambda \\ N u_{x} & \text { Nusselt number for non-rotating fully } \\ & \text { developed flow } \\ P r & \text { Prandtl number } \\ P r_{\epsilon}, P r_{k} & \text { Prandtl numbers for } \epsilon \text { and } \mathrm{k}, \text { respectively } \\ p & \text { Pressure } \\ R & \text { Mid-length radius of the duct } \\ R e & \text { Reynolds number } \\ R o & \text { Rotation number, } \omega \mathrm{d} / \mathrm{w}_{\text {in }} \\ R_{r} & \text { Root radius } \\ T & \text { Temperature } \\ T_{b} & \text { Bulk temperature } \\ T_{w} & \text { Wall temperature } \\ w & \text { Velocity vector } \\ \tilde{w}_{c} & \text { Centerline velocity } \\ w_{i n} & \text { Inlet velocity } \\ w_{x,}, w_{y}, w_{z} & \text { x-, y- and z-direction velocity components } \\ y+ & \text { Non-dimensional distance of near-wall cell } \\ & \text { center }\left(\delta \mathrm{C}_{\mu}^{1 / 4} \rho k^{1 / 2} / \mu\right) \\ z / d & \text { Non-dimensional axial distance from inlet, }(\mathrm{Z} \\ & \left.-\mathrm{R}_{\mathrm{r}}\right) / \mathrm{d} \\ & \end{array}$

\section{Greek Symbols}

$\nabla$

$\tilde{\Delta} T$

$\delta$

$\epsilon$

$\lambda$

$\mu$

$\mu_{t}$

$\rho$

$\underset{\sim}{\mathrm{i}} \partial / \partial \mathrm{x}+\underset{\sim}{\mathrm{j}} \partial / \partial \mathrm{y}+\underset{\sim}{\mathrm{k}} \partial / \partial \mathrm{z}$

Temperature difference, $T_{w}-T_{b}$

Distance of near-wall cell center from the wall

Dissipation rate of turbulent kinetic energy

Thermal conductivity

Laminar viscosity

Turbulent viscosity

Fluid density

Angular speed

\section{References}

Tekriwal, P., Heat Transfer Predictions with Extended k- $\epsilon$ Turbulence Model in Radial Cooling Ducts Rotating in Orthogonal Mode, ASME HTD-Vol. 226, Fundamentals and Applied Heat Transfer Research for Gas Turbine Engines, eds. D.E. Metzger and M.E. Crawford, pp. 41-50, 1992a. Also in ASME Journal of Heat Transfer, Vol. 116, pp. 369-380, 1994.

Yang, W.-J., Zhang, N., and Chiou, J., Local Heat Transfer in a Rotating Serpentine Flow Passage, ASME J. Heat Transfer, Vol. 114, pp. 354-361, 1992b.

Han, J.-C., and Zhang, Y.M., Effect of Uneven Wall Temperature on Local Heat Transfer in a Rotating Square Channel With Smooth Walls and Radial Outward Flow, ASME J. Heat Transfer, Vol. 114, pp. 850-858, 1992c

Wagner, J.H., Johnson, B.V., and Hajek, T.J., Heat Transfer in Rotating Passages with Smooth Walls and Radial Outward Flow, ASME Gas Turbine and Aeroengine Congress and Exposition, Paper 89-GT-272.

Morris, W.D., and Ghavami-Nasr, G., Heat Transfer Measurements in Rectangular Channels with Orthogonal Mode Rotation, ASME Gas Turbine and Aeroengine Congress and Exposition, Paper 90-GT-138. 
Lam, C.K.G., and Bremhorst, K.A., Modified Form of the k- $\epsilon$ Model for Predicting Wall Turbulence, J. Fluids Eng., Vol. 103, pp. 456-460, 1981

Patel, V.C., Rodi, W., and Scheuerer, G., Turbulence Models for Near-Wall and Low Reynolds Number Flows: A Review, AIAA J. Vol. 23, No. 9, pp. 1308-1319, 1985a.

Biswas, D., and Fukuyama, Y., Calculation of Transitional Boundary Layers with an Improved Low-Reynolds Number Version of the k- $\epsilon$ Turbulence Model, ASME Gas Turbine and Aeroengine Congress and
Exposition, Paper 93-GT-73.

Patankar, S.V., Numerical Heat Transfer and Fluid Flow, Hemisphere Publishing Corp., 1980.

Braaten, M., and Shyy, W., A Study of Recirculating Flow Computation Using Body-Fitted Coordinates: Consistency Aspects and Mesh Skewness, Numer. Heat Transfer, Vol. 9, pp. 559-574, 1986.

Shyy, W., Tong, S.S., and Correa, S.M., "Numerical Recirculating Flow Calculation Using a Body-Fitted Coordinate System," Numer. Heat Transfer, Vol. 8, pp. 99-113, 1985b. 

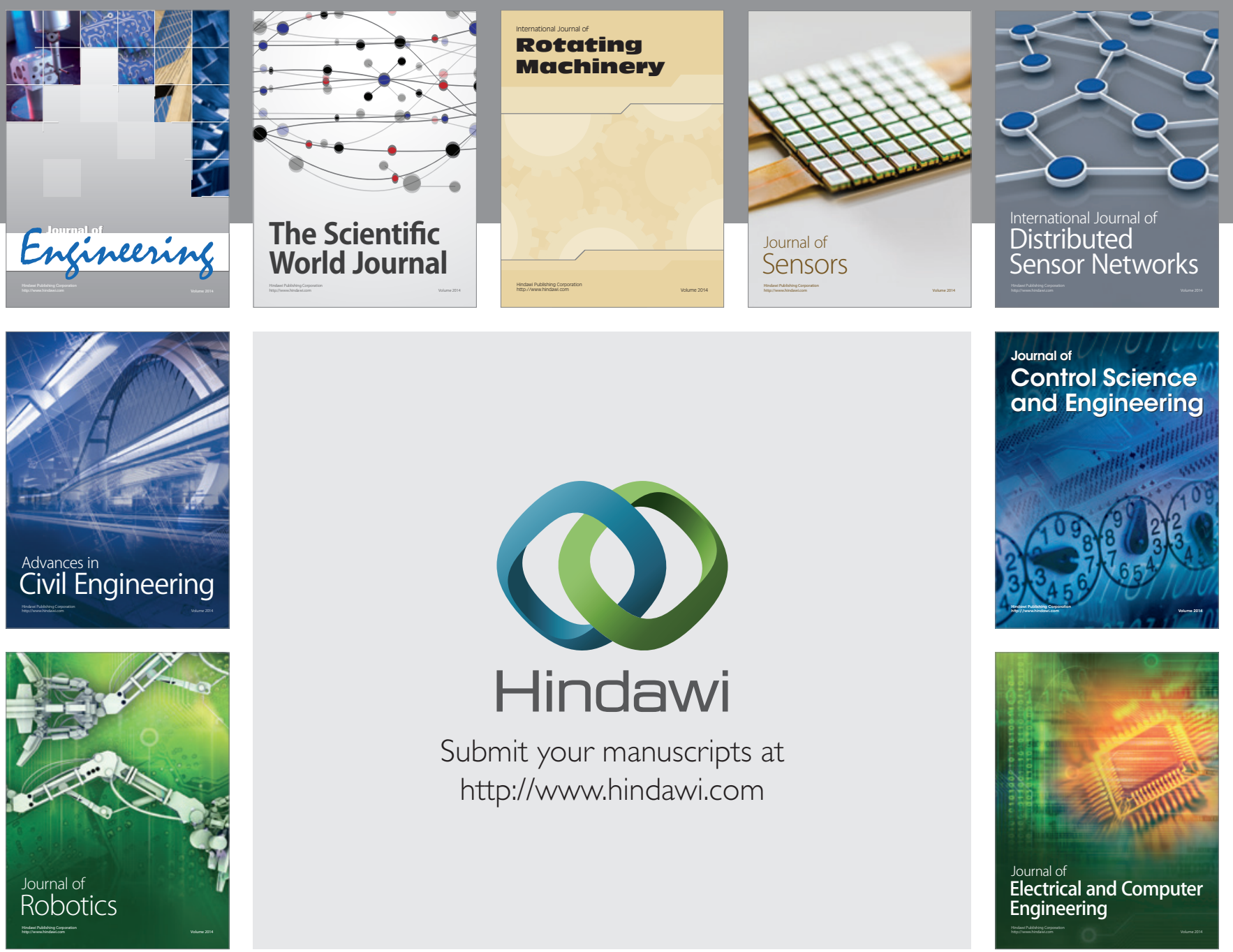

Submit your manuscripts at

http://www.hindawi.com
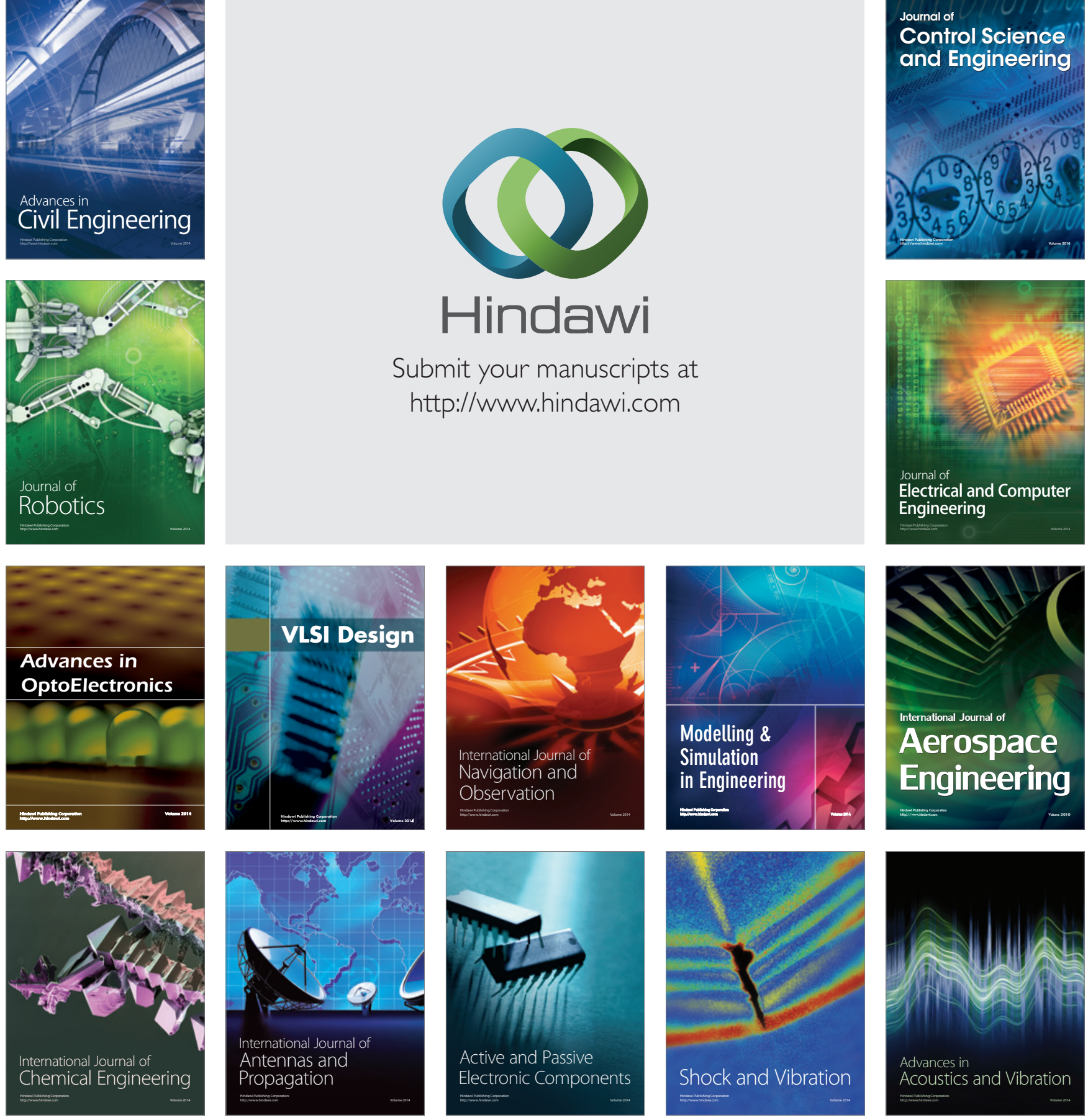